\title{
Social Recommendation and Visual Analysis on the TV
}

\author{
Cathal Gurrin*, Hyowon Lee, Paul Ferguson, \\ Alan F. Smeaton, Noel E. O'Connor \\ CLARITY: Centre for Sensor Web Technologies \\ Dublin City University \\ Dublin 9, IRELAND \\ *cgurrin@computing.dcu.ie
}

\author{
Yoonhee Choi, Heeseon Park \\ Media S/W Platform Lab. \\ DMC R\&D Center, Samsung Electronics \\ Gyeonggi-do 443-742, Korea
}

\begin{abstract}
In this paper, we present prototype interactive TV software that incorporates visual content analysis tools and social networking in the home TV. We present the challenges of working with the living room TV environment and outline how we have utilized visual processing and search technologies to address these challenges and create a novel prototype interactive TV system.
\end{abstract}

\section{Categories and Subject Descriptors}

H.3.3 [Information Storage and Retrieval]: Information Search and Retrieval - search process.

\section{General Terms}

Algorithms, Design, Human Factors.

\section{Keywords}

Content Analysis, Interactive TV, Multimedia Entertainment

\section{INTRODUCTION}

Increasing technological advancement means that it is now possible to store over a thousand hours of TV content on a Digital Video Recorder (DVR). How to provide effective interactive search and browsing functionality therefore becomes a challenging research topic. In a joint project between Dublin City University and Samsung Electronics, we set out to bridge the gap between academic research outputs and real-world interactive TV requirements, by implementing visual content analysis techniques and content search and recommendation to operate effectively in the relaxed environment of the living room TV. In this paper we report on the prototype software that we developed.

\subsection{REQUIREMENTS}

In defining this collaborative project, we specified a set of requirements for the interactive TV software to achieve. It must support social interaction among a social network of buddies; fast browsing of the entire video archive; smart segmentation and appropriate presentation of different content types (e.g. sports, news, movies); linking to related (internal and external) content; and not require any additional input than that afforded by the typical remote control that accompanies every TV.

\section{CHALLENGES OF INTERACTIVE TV}

The key challenges of developing for interactive TV are: limited

Copyright is held by the author/owner(s). MM'10, October 25-29, 2010, Firenze, Italy. ACM 978-1-60558-933-6/10/10. content search and manipulation capabilities by using a remote control as the interaction mechanism, distance from the screen, fixed resolution and operating in a distractive environment. Steady progress in the field of MultiMedia Information Retrieval (MMIR) allows us to address these challenges by providing a set of tools that could support new usage scenarios and features to enhance the user experience in today's digital media applications. Such tools include shot boundary detection and keyframe extraction, automatic metadata annotation and search, visual content analysis, content personalisation and recommendation and content summarization and novelty detection. We believe that careful application of such technologies can significantly enhance the TV viewing experience while avoiding complex interactions.

\section{TECHNICAL IMPLEMENTATION}

The prototype described in this paper has been implemented on a computer with a USB remote control and is displayed on a widescreen LCD TV. The remote control is the only input and content manipulation device. We used a computer with the understanding that the processing capabilities and storage will be supported by the TV or DVR. Keeping in mind the "lean-back" nature of TV interaction, very simple and shallow menu structure was devised which used the colour buttons on the remote control to invoke the core system functions when pressed (social interaction, browse/search within a show or within the archive, find similar content and EPG). In this way, we avoided relying on menu navigation which requires careful and focused user attention.

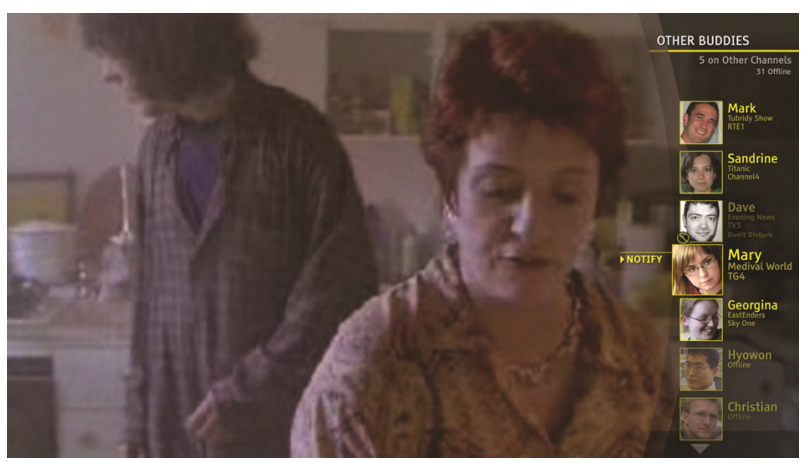

Figure 1. List of buddies to notify about a show.

Social Interaction - "Social TV" features are currently an active research topic with simple "presence alert" [1] and more recently design guidelines for social TV interaction [2] appearing. In our prototype, when a yellow button on the remote is pressed a list of the current user's buddies who are watching the same channel as the user appears from the right side of the screen (see Figure 1). 
The user can easily notify buddies about the current channel s/he is watching with simple up/down and OK buttons on the remote.

Browsing within a recorded TV show - pressing a blue button on the remote displays a visual content summary of the current show in the form of keyframes from identified scenes. This is achieved by employing a shot boundary detection algorithm [3] to segment the video into a sequence of shots, a keyframe extraction tool to extract a representative keyframe from every shot and a scene composition tool to concatenate adjacent shots into a logical scene unit for display. The user can then browse a TV show in a manner similar to the "chapter selection" feature of DVD movies. If needed, the user can examine all keyframes from the shots from that scene, providing a more find-grained level of browsing. This effectively makes the feature a 2-level hierarchical keyframe browser directly accessible with a TV remote control.

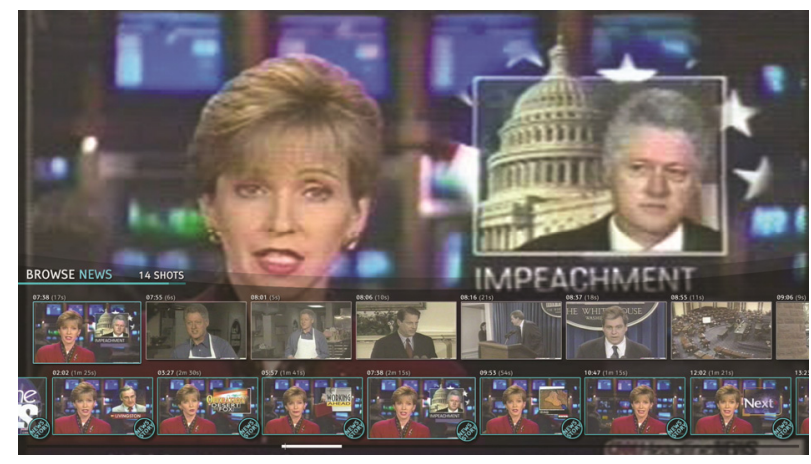

Figure 2. Browsing News Contents.

Depending on the genre of the show there are more appropriate ways to allow the user browse the content than simply presenting keyframes. For the three genres of news, sports and movies we developed and integrated tailored content analysis tools to support enhanced functionality. For example for TV news, we employed a news story segmentation algorithm similar to [4] to enable news story browsing (shown in Figure 2, the lower keyframes represent news stories and upper keyframes, shots from a selected story).

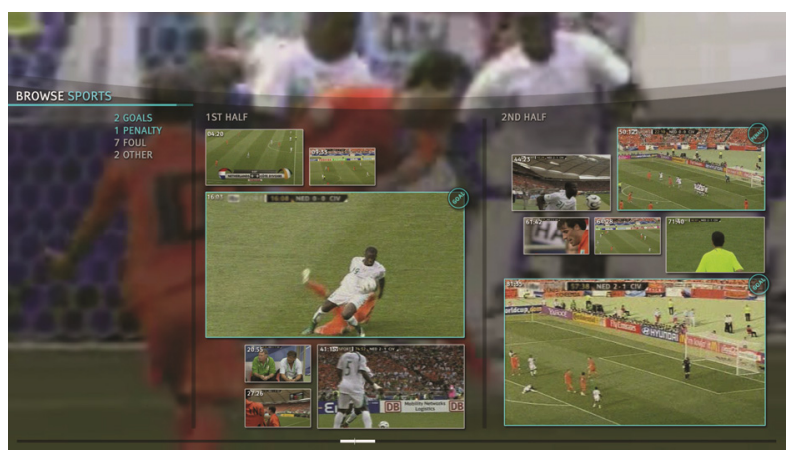

Figure 3. Browsing Sports Contents.

For sports shows we utilized field-sports event summarization [5] and on-screen layout tools segment a TV sports game (for a number of sports including soccer) into a set of events, identify the most important events and visually layout a game summary on the screen, with larger keyframes signifying the more important events of each half (as shown in Figure 3).

Finally, for movies, we utilize the shot boundary detection, keyframe extraction and scene composition tools to generate a
DVD-style chapter segmentation of the movie, which also supports shot-level browsing and linkage to related content.

Searching - while typing in a text-based query using a keyboard is common on the desktop PC and the WWW, such approaches for TVs and DVRs are cumbersome to use. Our approach was to support extensive content linkage by employing a combination of visual content-based similarity measures and metadata from EPG text to allow a feature called "Find Similar" when a user presses an orange button on the remote. Pressing this button slides in a panel showing other shows that are similar to the currently watched show, bypassing the need for a user input text query. A slide-in panel shows a selection of shows from the archive of recorded shows as well as video clips crawled from online sources. Furthermore, a simple tab organisation on the panel allows the user to easily find other video shots similar to the currently watched shot, letting the user jump from one part of a show to another part in a different show.

Other features include a red button, that when pressed, presents Electronic Program Guide where the user can see recommended shows and request recording, and a method for browsing the entire archive of recorded TV shows invoked by the green button.

\section{CONCLUSION}

The prototype described in this paper was developed as a prototype TV content management system implementing visual content analysis techniques and content search and recommendation techniques. The underlying tools were extensively tested and the whole prototype has been informally tested numerous times. Our preliminary findings indicate that potentially complicated multimedia functionalities can be successfully integrated into the TV in a straightforward and easyto-use manner. Future work includes evaluating the usefulness of additional content analysis tools that might enhance the TV viewing experience of a user in the living room TV environment.

\section{ACKNOWLEDGEMENTS}

We gratefully acknowledge the support of both Samsung Electronics, Korea and Science Foundation Ireland under grant number 07/CE/I1147.

\section{REFERENCES}

[1] T. Coates. Social software for set-top boxes. In Plasticbag.org, 2005.

[2] D. Geerts and D. De Grooff. Supporting the social uses of television: sociability heuristics for social TV. CHI 2009.

[3] Evaluating and Combining Digital Video Shot Boundary Detection Algorithms. Browne P, Smeaton A.F, Murphy N, O'Connor N, Marlow S and Berrut C. IMVIP 2000 - Irish Machine Vision and Image Processing Conference, Belfast, Northern Ireland, 31 August - 2 September 2000.

[4] N. O'Hare, A. Smeaton, C. Czirjek, N. O'Connor and N. Murphy. A Generic News Story Segmentation System and its Evaluation. ICASSP 2004, Montreal, Quebec, Canada, 17-21 May 2004.

[5] D. Sadlier and N. O'Connor. Event Detection in Field Sports Video using Audio-Visual Features and a Support Vector Machine. IEEE Trans. Circuits \& Sys. for Video Tech, 2005. 